\title{
Schweizerische Gesellschaft für Radioonkologie (SRO)
}

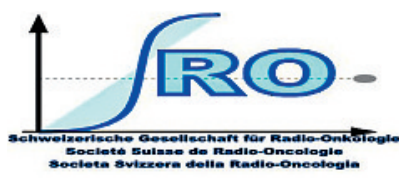

Günther Gruber

Präsident SRO

\section{Was ist Radioonkologie?}

Die Radioonkologie/Strahlentherapie befasst sich mit der medizinischen Anwendung von ionisierender Strahlung, um Krankheiten zu heilen bzw. um Beschwerden zu lindern. Die Bestrahlung erfolgt für den Grossteil der Patienten und Patientinnen aufgrund eines Malignoms. Mehrere Fachgruppen sind im Rahmen einer Strahlenbehandlung involviert: Neben dem Arzt sind dies Medizinphysiker, Medizinisch-technische Röntgenassistenten (MTRAs), Pflegepersonen und nicht zuletzt Techniker und Sekretariatspersonal.

\section{Die Schweizerische Gesellschaft für Radioonkologie}

Fachärztinnen und Fachärzte sind in der Regel Mitglied der SRO, der Schweizerischen Gesellschaft für Radioonkologie. Die Weiter- und Fortbildung ist ein zentraler Aufgabenbereich des Vorstands, der sich momentan aus fünf Mitgliedern zusammensetzt. In diesem sowie in anderen für die Radioonkologie/Strahlentherapie relevanten Punkten (z. B. Strahlenschutz, Qualitätssicherung) arbeitet die SRO eng mit der SASRO (Scientific Association of Swiss Radiation Oncology) und der SGSMP (Schweizerische Gesellschaft für Strahlenbiologie und Medizinische Physik) zusammen. Aktuell bestehen 15 Weiterbildungsstätten, 10 der Kategorie A (für 4 Jahre anerkannt) und 5 der Kategorie B (für 2 Jahre anerkannt), das Weiterbildungsprogramm zum Facharzt dauert 6 Jahre.

\section{Einige Zahlen}

Die SRO ist eine verhältnismässig kleine Fachgesellschaft. In den letzten 15 Jahren wurden lediglich 82 Facharzttitel von der FMH verliehen und dies, obwohl die Zahl der radiotherapeutisch behandelten Patienten jährlich steigt: Repräsentative statistische Zahlen zur Radioonkologie in der Schweiz zeigen: Im Jahr 2007 wurden schweizweit etwa 20000 Patientinnen und Patienten therapiert, davon etwa 800 aufgrund einer gutartigen Erkrankung (z. B. Epikondylitis, Arthrose). Im Vergleich zu 2004 entspricht dies einer Zunahme von $15 \%$. Die zunehmende Arbeitslast spiegelt sich auch insofern darin wider, dass 2007 pro Facharzt 236 Patienten behandelt wurden im Vergleich zu 202 Patienten im Jahre 2004. In

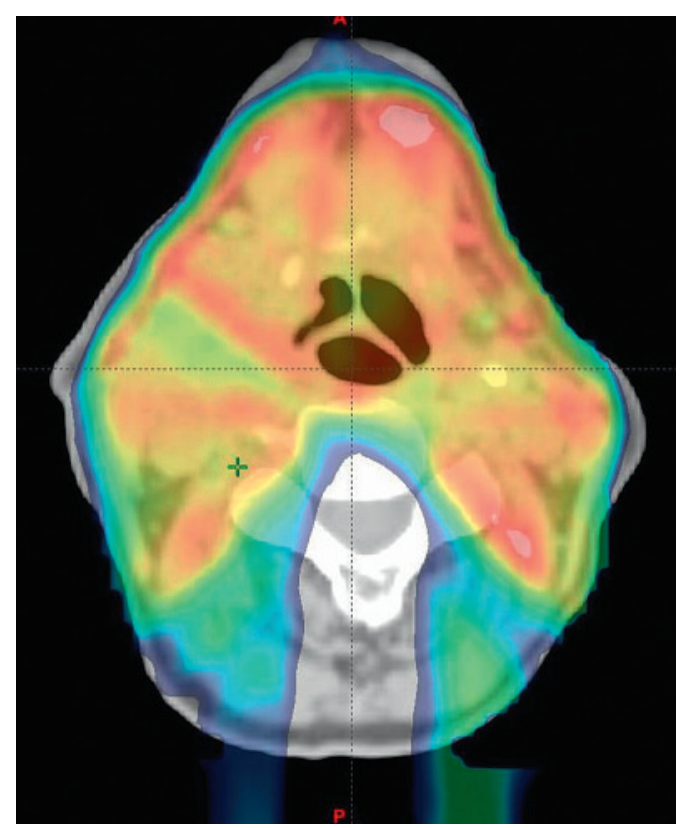

IMRT-Dosisverteilung.

den Niederlanden waren es 217 Patienten im Jahr 2007. Eindrücklich ist auch, dass sowohl die Anzahl der Hochvoltbehandlungssitzungen (2007: 371804) als auch der eingesetzten Bestrahlungsfelder (2007: 1333533) um etwa 40\% zugenommen hat, also überproportional im Vergleich zur Patientenzahl, was der zunehmenden Komplexität der Behandlungen Rechnung trägt. In der Tat stieg der Einsatz der Radiochirurgie um 21\%, der Brachytherapie (= Kurzdistanztherapie) um $86 \%$ und der intensitätsmodulierten Radiotherapie (IMRT) gar um 404\%! Im gleichen Zeitraum kam es aber zu einem leichten Rückgang der in der Strahlentherapie tätigen Ärzte (etwa -6\%). Einige Zentren haben Mühe, ihre Ausbildungsstellen zu belegen.

\section{Ausblick}

Es ist deshalb ein zentrales Anliegen der SRO, das Fach Radioonkologie insbesondere für junge Kolleginnen und Kollegen attraktiv zu machen. Mit der Integration neuer bildgebender Verfahren in den Behandlungsablauf (z. B. Bildfusion, Computertomogramm am Linearbeschleuniger), der Zunahme spezieller Applikationstechniken und der festen Einbindung der Radiotherapie in multimodale Therapiekonzepte sollte die Attraktivität des Faches aber gesichert sein. 